\title{
Translation of special texts from English into Russian: modern approaches to quality assessment
}

\author{
Ekaterina Kramnaya ${ }^{1 *}$, Rdouan Faizi $^{2}$, Maria Rudneva $^{3}$ \\ ${ }^{1}$ Yaroslavl State Technical University, 150023, Yaroslavl, Moskovskyi prospect, 88, Russian \\ Federation \\ ${ }^{2}$ ENSIAS Mohammed V University in Rabat, Avenue des Nations Unies, Agdal Rabat Maroc \\ B.P:8007.N.U, Morocco \\ ${ }^{3}$ RUDN University,6 Miklukho-Maklaya St, Moscow, 117198, Russian Federation
}

\begin{abstract}
Translation quality assessment is an urgent and complex problem, which is widely discussed by both theoretical researchers and practicing translators but today there is no single solution for this task. This article highlights and describes three approaches to assessing the quality of special translation: scientific, normative, including standards as well as practical guides, and formalized. The author demonstrates synergetic effect of combination of these three approaches.
\end{abstract}

\section{Introduction}

Quality assessment of special translation is a complex problem which is widely discussed by both theoretical researchers and practicing translators. An efficient assessment system is necessary for all participants of the process: clients, translators, editors; it is also relevant for organizing the process of teaching special translation, in which teachers and students participate. Today there is no single solution for this task as they have to take into account the variety of special texts and options for their presentation, different needs the translated text to suit and approaches to translation process. Also, there cannot but point the critical importance of translation quality for its final recipient. The purpose of this work is to characterize modern practical and scientific approaches to assessing the quality of special translation in order to identify the ideas that connect them and create a system for their presentation.

\section{Methods and materials}

\subsection{Special translation}

This article deals with the assessment of quality of special translation. The term "special translation" refers to the translation of texts related to a particular specialty and operating with highly specialized terminology. At the same time, the range of special technical texts is rather wide: technical documentation, business documents, articles and monographs, regulatory documents, and many others. Special translation is done for practical purposes and, therefore, the result of this process should be viewed as a finished product, ready to use and from this point of view there should be special requirements to the translator: they must

\footnotetext{
*Corresponding author: kramnaya@yandex.ru
} 
not only accurately convey the content of the original text, but take into account the compliance with the rules and norm of the target language, apply proper professional terminology, meet the requirements for text design, keep proper metric system, work through not only text but also graphical information.

Now consider the above-described concept with an example of translating an instruction on equipment installation. This is a critical document for the end user, and the issue of quality translation is really vital here. To translate instructions, it is not enough to know a foreign language and translation techniques - you have to know the subject and have clear understanding of the practice described in the instructions. Only in this case, the translator will be able to use the correct terminology and talk to the professionals, the end users of the translation, in the same language. For translation from English into Russian, the user will use reference books and national standards to define terms, but this search also requires some basic knowledge. The next important point is to comply with the norms of the translation language. This includes both spelling and punctuation features, as well as the neutral style of presentation typical of Russian instructions with impersonal predicative constructions and imperative verbs instead of personal forms. Attention should also be paid to the transfer of metric and non-metric measures, taking into account the specifics of each specialty. Although as a rule in the Russian-language technical literature it is widely recommended to use the International system of units (SI) when specifying dimensions and other things, the diameters of pipes and some fasteners must be specified in inches. We should also note the processing of non-text information: instructions of this type often contain a large number of drawings, Figures, diagrams, etc. The translator must not only "read" this information and relate it to the text content, but also make it understandable to the user in the target language. Besides, proper text design should be considered. Often, the translator takes the issue of correct text formatting and design of its individual elements too easy, it is especially true for students and translators who have not previously dealt with technical documentation. However, standard text markup allows users quickly navigate through a large text, and correctly highlighted words, such as DANGER or WARNING, attract attention and help to avoid trouble. Moreover, the execution of such documents is subject to national and international standards. Today, many documents are transferred to electronic format, and the system of sections and paragraphs also turns into a system of hyperlinks, so the requirements for clear, logical and uniform design are increasing.

The general relationship between the components of special text translation can be presented as follows: 
TRANSLATION OF SPECIAL TEXT (INSTRUCTION ON EQUIPMENT INSTALLATION)

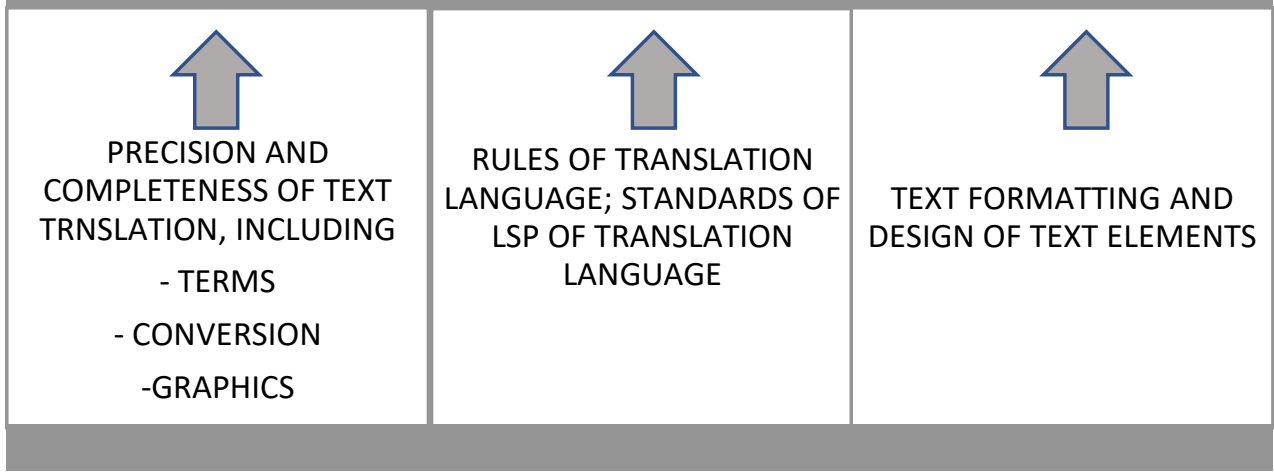

Fig. 1. Components of special text translation.

This example shows how many factors should be taken into account both when translating a special text and when assessing it.

Based on these features, B. N. Klimzo [2] gives the following practical classification of translation quality:

- dictionary translation, "performed only based on dictionaries", when other sources of information are not available to the translator

- a decent translation that "does not contain errors in the essence of the source text, distortions of the author's thoughts, translation gibberish and is written in compliance with the grammatical and stylistic norms of the translation language»

- a full-fledged translation in which "all the requirements for a good translation are met and in addition, extra work is performed to reasonably eliminate errors and alogisms, build equivalents of terms that are not in the dictionary, and converse dimensions»)

All three types of translation in this classification imply the most conscientious attitude to work on the part of the performer. Here, the quality of translation is determined by the availability of the translator's information base and the knowledge of the subject area, but the degree of responsibility of the translation approach is not reflected. In different situations, the customer or translation end user may find any of the above types sufficient and recognize its quality as satisfactory, but this assessment, which is outwardly subjective, will be based on a more complex combination of factors.

\subsection{Approaches to assessing the quality of translation and criteria for their suitability}

As noted above, today there is no universal system for evaluating the quality of translation, but three relevant approaches are applicable to solving this problem:

- scientific, which involves the work of translation researchers dedicated to quality assessment;

- regulatory, including industry standards and practical recommendations

- formalized approach as a mathematical solution to the problem

Taking into account the specifics of the translation of a special text, these approaches will be evaluated in terms of their objectivity, completeness, consideration of the solution of individual matters, and consideration of the design of the finished translation. Let's explain some of these criteria. The objectivity of the assessment means its impartiality and validity. 
Completeness refers to the assessment of the correspondence between the content of the translation text and the original text and their communication functions as a whole, and not by individual fragments. Consideration the solution of individual matters means evaluating various specific features of the translation of a special text: from the transfer of proper names to the presentation of mathematical formulas.

\subsection{Scientific approach}

The scientific vision of the problem of translation quality assessment is described in the works by V. N. Komissarov [3], A. D. Schweitzer [7], V. V. Sdobnikov [5] and many other well-known Russian translation theorists. The main objects of research in their articles and monographs are the concepts of equivalence and adequacy. Thus, V. N. Komissarov defines translation equivalence as "the consonance of the content in the original text and the translation" [3]. The author continues that the equivalence criterion is not rigid and it is possible to determine "the maximum possible semantic proximity of multilingual texts, as well as to determine the minimum proximity to the original, at which this text can be recognized as an equivalent translation" [3]. He identifies several levels of translation equivalence, that is, the semantic proximity of the texts in source language and in translation language, which are determined by a specific communicative context. Commenting on this approach, V. Sdobnikov emphasizes that in such a system, "equivalence relations are established at the level that is necessary and sufficient for the implementation of the communication goal." And concludes that "the choice of the equivalence level ... is determined by the task of achieving translation adequacy. That is, equivalence turns out to be a category subordinate to adequacy, and it is secondary in its significance. " [5] A.D. Schweitzer believes that "if equivalence answers the question of whether the translated text identical to the original, then adequacy answers the question of whether translation as a process complies with particular communicative conditions" [7]. Analyzing the current interpretations of these two concepts, V.V. Sdobnikov asserts that "for each type of translation, there are different ways of correlating adequacy and equivalence " and if the latter can be determined to different degrees, then there are only two categories of adequacy: the translation may be adequate or not adequate. As can be seen from the above points of view, researchers interpret these two concepts quite similarly, but there is no consensus. It should also be noted that despite profound development of criteria and levels, equivalence and adequacy remain subjective. They should be the basis of the assessment, but they cannot be the only ones that determine the quality of translation of special texts.

\subsection{Normative approach}

The normative approach in this article refers to the use of various industry regulatory documents, as well as guides, recommendations, and other developments of practicing translators and organizations focused on the requirements of final recipients. Industry standards, both international and Russian, assume that a quality product can be obtained only if the production process is properly organized. Thus, the international standard "ASTM F2575-14" is intended "... to promote clear understanding [by the parties to the translation process] in order to avoid potential waste of time, money and quality in the project "[8], and the standard "ISO 17100: 2015" defines requirements for all aspects of translation that directly affect the quality and delivery of translation services " [9]. In 2014, the Russian "GOST R EN 15038-2014" was approved and it is identical to another European regulation "EN 15038: 2006 Services to the population - Translation services - Service requirements". It also focuses on the organization of the translation process and the relationship between the translator (translation service provider) and the translation client. 
The authors of the "Rules for Translation and Special Types of Linguistic Services", which were adopted in 2014 and are also an official regulatory document, tried to solve the problem of assessing the quality of translated text [4]. Translation quality criteria include: compliance with the customer's requirements and compliance with the source text in terms of content, meaning, and design; no grammatical, spelling, or punctuation errors; compliance of the translation terminology with the industry affiliation of the source text; uniformity of terms, names, symbols, abbreviations, symbols [5]. It is obvious that the presented criteria combine both subjective assessment and objective subject requirements, but the latter seem to be very random and general.

The methodological recommendations developed by practicing translators as well as by the organizations providing translation services and professional associations also contribute to formation of a quality assessment system. They crystallize all the matters occurring in written translation practice and conventional norms of translation for specific purpose into practical guidelines. First of all, it is worth noting "Translation - Recommendations to the Translator, the Customer and the Editor" (hereinafter-Recommendations), the Third edition of which was approved in 2015 at the Translation Forum Russia [1]. The Recommendations is not an official guidance. The developers call this document a "reference manual" and explain that "Recommendations can be called a standard to the extent that they are accepted by the participants of the market and acquire the norms of business custom." [1]. The Recommendations include the Main Part and Supplementary part. The Main part, besides describing the translation process from the moment of selecting a translator to commissioning the project, sets out detailed requirements for the quality of the translation text as well as for the quality of original text, both design and content. The developers point out that these requirements are based on experience and expertise of many professional translators and related business. The Supplementary part containing Appendices to the Recommendations was developed by different authors and relate to a variety of matters that arise in the practice of a translator: from a sample $\mathrm{CV}$ of a freelance translator to transcription systems for the Korean language. Especially worth noting is the translator's detailed checklist, which can be used by all interested parties of the translation project. Today, many translation entities use the Recommendations as a basis for developing their own quality standards.

\subsection{Formalized approach}

A formalized approach to quality assessment of special translation is an innovative solution dictated by the introduction of digital technologies in the process of information exchange and processing, including translation. It consists in a mathematical description of the translation process and comparison of the texts in source language and translation language. The most developed models to date are described in the "Rules for Translation and Special Types of Linguistic Services" [4] and in Appendix 17 "Translation - Recommendations to the Translator, the Customer and the Editor" [5]. They use the TQI (Translation Quality Index), which takes into account the number of errors and their significance. Each error is evaluated in points and then, according to a certain formula, the quality index is calculated in order to make further decision on the suitability of the translation. The effectiveness of this method depends on the elaboration of evaluation criteria and the qualification of experts. At the same time, there are ready-made classifiers, for example, the international SAE J2450, which is used to evaluate translation in the automotive industry. The "Rules ..." provides a more universal classification of translation errors by severity and by group. All errors are divided into insignificant, significant, gross and occasional, and the decision on the suitability of the translation is proposed to be made taking into account the ratio of the received TQI and the volume of the translation performed [4]. Obviously, this approach is objective, but it is quite time-consuming, especially at the stage of identifying and marking errors, if this work 
is done manually. However, its further development is rather prospective and in the future it will result in automation of the quality assessment process, which is possible for working with certain types of special text, such as instructions, technical documentation, etc.

\section{Results and discussion}

The scientific approach allows you to evaluate the quality of translation in General. Basic criteria such as equivalence and adequacy help to make a conclusion about how complete the translation is and how functional it is. At the same time, the conclusion about the adequacy of the translation and the equivalence of the contents of the translation texts and the original may be based on a subjective opinion and may be challenged. The normative approach is aimed at solving the problems of organizing the translation process, but it also takes into account working with special elements of technical translation, such as dimensions, notation, etc. Applicable to such individual aspects, the normative approach is fairly objective, since the recommendations and standards contain clear criteria that leave no room for reservations. The formalized approach with mathematical processing of translator's errors may become the most objective and it will be able to fully assess the quality of translation of special texts, but at the moment the main drawback/ is its complexity, as well as the inability to evaluate the functionality of the translation.

\section{Conclusion}

To sum, despite the efforts of researchers and the professional community, the question of effective criteria for evaluating the quality of translation remains open. Even taking into account the specifics of technical texts, the evaluation basis consists of equivalence and adequacy criteria. Proper organization of the translation process, including compliance with clearly defined requirements for the texts of the source language and translation language, will allow to get a high-quality translation that is suitable for use in a particular industry. A carefully configured formalized assessment allows to describe one's attitude to the result of the translation process impartially and avoid conflict situations. All these approaches are necessary. The combination of all three approaches can have a synergistic effect, and for this purpose, the quality assessment of special translation should be given sufficient attention at the stage of training translators in the field of professional communication.

\section{Acknowledgments}

This work was supported by the Russian Foundation for Basic Research, grant No. 17-3410303

This paper was financially supported by the Russian Foundation for Basic Research, grant No. 20-012-22046.

\section{References}

1. N.V. Duplensky, Written translation. Recommendations to the translator, customer, and editor. 3rd edition, Moscow (2015)

2. B.N. Klimzo, The Craft of a Technical Translator, pp.454-455, Moscow (2007) 
3. V.N. Komissarov, Translation theory - linguistic aspects Moscow (1990)

4. PR. 50. 1. 027-2014 "Rules for Translation and Special Types of Linguistic Services". Federal Agency for technical regulation and Metrology-Moscow: STANDARTINFORM (2014)

5. V.V. Sdobnikov, O.I Petrova Translation theory, pp. 190-193 (AST: East-West, Moscow 2007)

6. D. Tishin, Techniques for formalized assessment of translation quality, in Written translation. Recommendations to the translator, customer, and editor-3rd edition-Appendix 17, Moscow (2015)

7. A.D Schweitzer, Theory of translation: Status, problems, and aspects, pp. 92-99 (Nauka, Moscow, 1988)

8. ASTM F2575 - 14 Standard Guide for Quality Assurance in Translation https://www.astm.org/Standards/F2575.htm

9. ISO 17100: 2015Translation services - Requirements for translation services. Retrieved from: https: / / www. iso. org/obp/ui/ \# iso:std:iso:17100:ed-1:v1: 